\title{
DOSSIER
}

\section{O pé dos diabéticos}

GEMA PONCE REVILLA*, ARMANDO BRITO DE SÁ**, JOÃO SEQUEIRA CARLOS***

\section{RESUMO}

Introduç̃o: As lesões nos pés dos diabéticos representam um aspecto crítico dos cuidados de saúde, quer sua pela frequência quer pelas consequências da doença. Pretende-se, neste trabalho, estabelecer orientações práticas para a abordagem deste problema nos cuidados primários.

Metodologia: Pesquisa nas bases de dados Pubmed e fontes documentais de referência. Conclusões: Ressalita a importância da avaliação periódica dos pés do diabético, para a estratificação do risco de lesões, a prevenção de úlceras e o tratamento atempado e adequado das mesmas.

Palavras-Chave: Pé Diabético; Pé Neuropático; Pé Isquémico; Úlcera do Pé Diabético; Estratificação do Grau de Risco; Seguimento do Pé Diabético; Prevenção.
*Interna do $3^{\circ}$ ano de Clínica Geral, USF Rodrigues Miguéis Centro de Saúde Benfica **Médico de Família, USF Rodrigues Miguéis - Centro de Saúde Benfica ***Médico de Família, Unidade Medicina Familiar Hospital da LuZ

\section{INTRODUÇ̃̃o}

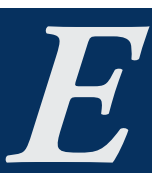

stima-se que em Portugal existam 500.000 diabéticos, $15 \%$ dos quais apresentando condições favoráveis ao aparecimento de lesões nos pés. Em Portugal, a diabetes é causa de 40-60\% das amputações efectuadas por causas não traumáticas (aproximadamente 500 amputações/ano). ${ }^{1-3} \mathrm{O}$ "pé diabético" é, portanto, uma das complicações mais graves da diabetes. ${ }^{4}$ Mas o que é o "pé diabético»?

Em 1955, Lowrie publicou o primeiro artigo intitulado "The diabetic foot", mas só nas últimas décadas o termo "pé diabético" se tornou comum. ${ }^{5}$ Hoje, 52 anos depois, o uso disseminado da expressão tem vantagens e desvantagens. A sua inespecificidade, por englobar distúrbios de qualquer gravidade, tecido e arquitectura do pé, é a principal desvantagem. Entre as vantagens da sua popularização está a ênfase no exame do pé (quase sempre negligenciado no exame médico) e a profilaxia das lesões. ${ }^{6}$

No consenso publicado pela Sociedad Española de Angiología y Cirugía Vascular $^{7}$, o pé diabético é definido como una «alteração clínica de base etiopatogénica neuropática e induzida pela hiperglicemia mantida, com ou sem coexistência de isquemia, e prévio desen- cadeante traumático, produzindo lesão e/ou ulceração do pé». Isto implica que os pés dos doentes com diabetes sem lesões e/ou úlceras ficam fora da definição do pé diabético? Perante esta dúvida, parece conveniente falar do pé do diabético, em detrimento de pé diabético, já que os pés do diabético, incluindo aqueles sem "pé diabético", devem ser alvo de atenção nos cuidados de saúde primários. Esta revisão tem como objectivo a elaboração de um guia prático que ajude na compreensão, seguimento e tratamento desta afecção.

\section{Métodos}

Foi realizada uma pesquisa na base de dados Medline/Pubmed. Não foram colocados limites temporais à pesquisa. Foram utilizadas as seguintes palavras-chave: pé diabético, pie diabético, diabetic foot care, risk and diabetic foot, ulcerated foot, prevention of diabetic foot. Foram seleccionados todos os artigos cujos título ou resumo foram considerados relevantes no contexto da revisão em causa. Realizou-se, ainda, pesquisa documental em obras de referência, orientações da Direcção Geral de Saúde (DGS) e material didáctico da Associação Protectora dos Diabéticos Portugueses.

\section{FISIOPATOLOGIA DO PÉ DO DIABÉtICO}

A triade, composta por neuropatia, doença arterial periférica (DAP) e infecção, constitui a base das alterações no pé dos diabéticos.

A neuropatia diabética (periférica e autonómica) desempenha um papel central. ${ }^{4} \mathrm{~A}$ incidência de neuropatia di- 
abética aumenta com a idade do doente, o tempo de duração da diabetes e com a gravidade da hiperglicemia. A perda da sensibilidade sensitiva origina insensibilidade do pé e úlceras por microtraumatismos que passam despercebidos. As sensibilidades vibratória e proprioceptiva são atingidas antes da táctil, álgica e térmica, donde a importância de testar as duas primeiras. A neuropatia motora do diabético atinge fundamentalmente os músculos interósseos (surgindo dedos em gatilho ou martelo, diminuição da almofada plantar e subsequente pressão anómala e aumentada sob a cabeça dos metatársicos) e menos frequentemente o músculo tibial anterior, o que desencadeia pé pendente e aumento da pressão na área posterior do pé. A disfunção das fibras nervosas simpáticas do sistema autonómico provoca perda do tónus constritor ao nível das artérias e arteríolas e disfunção das glândulas sudoriparas. A pele fica quente, desidratada, espessa e fissurada, com ingurgitamento venoso e aumento da incidência de osteopénia, fracturas espontâneas e neuroartropatia de Charcot.

A doença arterial periférica (DAP) tem também um papel importante no pé dos diabéticos, sendo o factor prognóstico mais importante numa úlcera do pé. A DAP caracteriza-se pela presença de aterosclerose (que provoca isquémia por estenose e obstrução arterial) e calcificação da túnica média, produzindo um vaso rígido sem estreitamento do lúmen. O diabético com DAP pode estar assintomático, sofrer claudicação intermitente ou, em casos mais graves, ter dor isquémica em repouso, úlceras e gangrena. Frequentemente, os doentes com DAP não têm sintomas devido à perda de sensibilidade causada pela neuropatia periférica coexistente.

Os microtraumatismos, associados à susceptibilidade à infecção, provocada pelo ambiente hiperglicémico, a disfunção leucocitária e da imunidade ce-

\begin{tabular}{|c|c|}
\hline \multicolumn{2}{|c|}{ QUADRO I } \\
\hline CLASSIFICAC̣ÃO FISIOPATOL & D́GICA DO PÉ DIABÉTICO \\
\hline Pé Neuropático & Pé Isquémico \\
\hline Quente & Frio \\
\hline Rosado & $\begin{array}{l}\text { Pálido com a elevação, } \\
\text { cianosado com o declive }\end{array}$ \\
\hline Pele seca e fissurada & Pele fina e brilhante \\
\hline Deformações & - \\
\hline Insensível à dor & Com sensação dolorosa \\
\hline Pulsos amplos & $\begin{array}{l}\text { Pulsos diminuídos ou } \\
\text { ausentes }\end{array}$ \\
\hline Veias ingurgitadas & $\begin{array}{l}\text { Aumento do tempo } \\
\text { de enchimento capilar }\end{array}$ \\
\hline Edemaciado & Sem edema \\
\hline $\begin{array}{l}\text { Se úlceras: } 1^{\circ} \text { e } 5^{\circ} \\
\text { metacárpico e calcâneo } \\
\text { (posterior). Redondas, } \\
\text { com anel queratósico } \\
\text { periulcerativo. Não dolorosas }\end{array}$ & $\begin{array}{l}\text { Se úlceras: látero-digital. } \\
\text { Sem anel queratósico. } \\
\text { Dolorosas }\end{array}$ \\
\hline
\end{tabular}

lular e a pele seca, são dois factores importantes na fisiopatologia.

Classicamente, são definidos três tipos de pés: o pé neuropático, o pé isquémico e o pé neuroisquémico. Dois terços das lesões aparecem em pés predominantemente neuropáticos. No Quadro 1 mostram-se, resumidamente, os sintomas e sinais que ajudam ao diagnóstico diferencial de cada uma destas entidades.

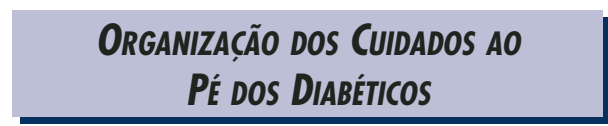

Segundo o grupo de Trabalho Internacional Sobre o Pé Diabético, ${ }^{8}$ é necessária uma estrutura global bem organizada e com uma comunicação fluida, para satisfazer as necessidades de cuidados dos pés dos diabéticos. Para isso é necessária a criação de uma equipa multidisciplinar que abarque pelo menos três níveis de controlo do pé:4,8

\section{Nivel I}

São objectivos prioritários, neste nível 
de cuidados, o reforço da educação do doente e familiares, os cuidados de lesões não ulcerosas, o tratamento de úlceras superficiais e o seguimento da patologia ulcerosa já referenciada a centros mais diferenciados. A equipa deverá ser constituída por um médico de família, enfermeira especializada em diabetes e um podologista.

\section{Nivel II}

São objectivos deste nível de cuidados a avaliação dos doentes com patologia ulcerosa, isquémica e com patologia ulcerosa complicada por infecção e/ou necrose, a necessitar de eventuais desbridamento cirúrgico e/ou internamento. A equipa deverá ser constituída por médico endocrinologista ou internista, ortopedista ou cirurgião geral e enfermeiro e/ou profissional treinado em podologia.

\section{Nivel III}

São objectivos deste nível de cuidados a avaliação das úlceras graves, assim como a identificação da necessidade de investigação vascular. A equipa deverá ser constituída por médico endocrinologista ou internista, cirurgião, cirurgião vascular, fisiatra, enfermeiro, profissional treinado em podologia e técnico de ortóteses.

\section{Avallaç̃o Periódica dos Pés do Diabético: Estratificaç̃̃o do RISCO E SEGUIMENTO}

Todos os diabéticos devem ser avaliados periodicamente, com o objectivo de identificar o pé em risco de ulceração e os pés com úlceras activas. ${ }^{4,9,10}$ Após a identificação dos factores de risco (Quadro 2) podem-se agrupar os diabéticos segundo categorias, o que ajuda a estabelecer um esquema de seguimento adequado. No Quadro 3 descreve-se a avaliação periódica dos pés dos diabéticos, baseada na Circular Normativa de

\section{QUADRO II}

\section{FACTORES DE RISCO NOS PÉS DOS DIABÉTICOS}

Úlcera ou amputação prévias
Complicações tardias da diabetes
Diminuicão da acuidade visual
Desconhecimento dos riscos da doença
Condições socioeconómicas deficientes
Depressão
Pele seca do pé
Presença de calosidades, gretas ou onicomicose
Presença de edema
Deformidade dos dedos ou rigidez articular
Neuropatia
Doença arterial periférica
Uso inadequado de meias e calçado

2001, da DGS. ${ }^{4}$

A avaliação dos factores de risco implica a realização da história clínica e do exame objectivo: 8,10

- Na história clínica deve-se incluir o contacto do diabético (telefone de contacto e morada), os antecedentes pessoais (incluindo os hábitos de vida, como tabagismo, etilismo e exercício habitual, o tipo de diabetes e ano de diagnóstico, história de nefropatia, retinopatia, úlcera anterior/amputação e presença de doenças concomitantes) e o tratamento actual. É necessário investigar a presença de sintomas, como parestesias, dor em repouso, claudicação e cansaço ou diminuição da velocidade da marcha. De realçar que a dor em repouso e a claudicação podem estar ausentes em presença de neuropatia concomitante. ${ }^{11}$

- O exame objectivo deve realizar-se com o doente descalço, inicialmente de pé e depois em decúbito. ${ }^{12}$ Serão avaliados:

- Pele e faneras (cor, temperatura, hidratação da pele, distribuição pilosa, presença de edema, calosidades, fissuras, maceração interdigital, úlceras, amputações, alterações das unhas).

- Sistema osteoarticular (deformações, proeminências ósseas, mobilidade arti- 


\section{DOSSIER}

ESQUEMA DE SEGUIMENTO DOS PÉS DIABÉTICOS

\begin{tabular}{|l|l|l|}
\hline \multicolumn{1}{c|}{ Pé } & \multicolumn{1}{|c|}{ Factores de risco } & \multicolumn{1}{c|}{ Esquema de seguimento } \\
\hline Baixo risco & Ausência de factores de risco & Anual \\
\hline Médio risco & $\begin{array}{l}\text { Um ou mais factores de risco (excepto neuropatia, doença } \\
\text { arterial periférica, deformação, ulceração ou amputação prévias) }\end{array}$ & Semestral \\
\hline Alto risco & $\begin{array}{l}\text { Neuropatia, doença arterial periférica, deformação, } \\
\text { ulceração, amputação prévias }\end{array}$ & Mensal/trimestral \\
\hline
\end{tabular}

cular)

- Sistema vascular (pulso tibial posterior e pedioso, índice sistólico tornozelo-braço - ITB). ${ }^{13}$

O ITB deve realizar-se em todos os diabéticos com mais de 50 anos, diabéticos com menos de 50 anos, mas com um ou mais factores de risco, diabéticos com mais de dez anos de diagnóstico e diabéticos com sintomas sugestivos de DAP. Se o teste não mostra obstrução repete-se de cinco em cinco anos. Se aparecer obstrução moderada ou severa ou se o teste for negativo, mas houver uma forte suspeita clínica de doença arterial periférica, deve-se realizar um estudo vascular mais completo. Os valores de referência do índice estão indicados no Quadro 4.

- Sensibilidade e reflexos: avaliam-se a percepção da pressão (com o monofilamento de Semmes-Weinstein), a sensibilidade vibratória (com o diapasão de $128 \mathrm{~Hz}$ ), a discriminação (com picada de alfinete no dorso do pé) e a sensibilidade superficial (com algodão no dor-

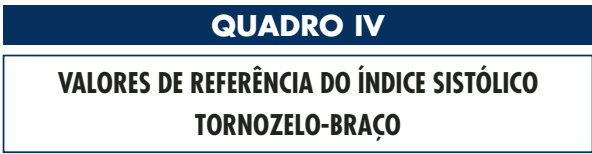

\begin{tabular}{|l|l}
\hline Valor & Grau de obstrução \\
\hline $0,91-1,30$ & Sem obstrução \\
\hline $0,70-0,90$ & Obstrução ligeira \\
\hline $0,40-0,69$ & Obstrução moderada \\
\hline$<0,40$ & Obstrução severa
\end{tabular}

so do pé). Avaliam-se, ainda, os reflexos aquilianos.

- Avaliação do calçado e meias: observam-se o material das meias e a presença de costuras e/ou remendos. Avalia-se o material do sapato, a integridade da sola, a altura do dorso do sapato, a altura do salto, a presença de palmilha, a firmeza do calcanhar, a biqueira e a presença de corpos estranhos.

\section{Tratamento dos Pés dos Diabéticos}

Existem diferentes escalas de classificação das úlceras no "pé diabético", ${ }^{14}$ sendo a classificação de Wagner a classificação mais frequentemente utilizada. ${ }^{15}$ Com base nela, foi elaborado o esquema apresentado no Quadro 5, ${ }^{8,15}$ útil na hora de decidir sobre o adequado seguimento e/ou tratamento dos pés dos diabéticos.

\section{Grau 0 - Pé do diabético com baixo risco de úlcera}

Neste grau, o papel dos cuidados primários de saúde assenta no reforço positivo $^{9}$ e na prevenção, com o ensino de cuidados (unhas, hidratação da pele, prevenção das micoses, calçado adequado, mobilização) e a recomendação de hábitos de vida saudáveis (alimentação adequada, caminhar diariamente meia hora, nadar, andar de bicicleta, ${ }^{12}$ actividade física com os pés, ${ }^{16}$ redução do álcool, cessação tabágica).3,17 Como em todos os diabéticos o bom 


\begin{tabular}{|c|c|c|}
\hline \multicolumn{3}{|c|}{ QUADRO V } \\
\hline Grau & Lesão & Características \\
\hline 0 & «Pé com risco baixo de úlcera» & Ausência de factores de risco \\
\hline 1 & «Pé com médio/alto risco de úlcera» & $\begin{array}{l}\text { Calosidades, fissuras, cabeças metatársicas proeminentes, } \\
\text { dedos em garra, outras anormalidades ósseas } \\
\text { Neuropatia diabética } \\
\text { Doença arterial periférica } \\
\text { Úlcera prévia ou amputação }\end{array}$ \\
\hline 2 & $\begin{array}{l}\text { Úlcera superficial sem infecção } \\
\text { evidente }\end{array}$ & Destruição total da pele \\
\hline 3 & Úlcera profunda & $\begin{array}{l}\text { Penetra pele, gordura, ligamentos } \\
\text { Não penetra o osso } \\
\text { Infecção do pé: sinais de infecção (febre, leucocitose), } \\
\text { secreção purulenta, ou dois ou mais sintomas locais } \\
\text { (vermelho, quente, doloroso, crepitação) }\end{array}$ \\
\hline 4 & $\begin{array}{l}\text { Úlcera profunda com celulite, } \\
\text { abcesso, ou envolvimento ósseo }\end{array}$ & Extensa e profunda, secreç̃̃o e mau cheiro \\
\hline 5 & Gangrena localizada & Necrose de uma parte do pé: planta, calcanhar, dedos \\
\hline 6 & Gangrena extensa & $\begin{array}{l}\text { Atinge todo o pé } \\
\text { Sintomas e sinais sistémicos }\end{array}$ \\
\hline
\end{tabular}

controlo metabólico e das doenças concomitantes são tarefas essenciais. ${ }^{9}$

A avaliação dos pés deve ser efectuada anualmente e como descrito anteriormente.

\section{Grau 1 - Pé do diabético com risco médio e alto de úlcera}

Além das medidas anteriores, devem-se tratar as patologias específicas do pé com médio/alto risco de úlcera. A hiperqueratose pode ser tratada com pomada de ureia (duas vezes por dia durante sete dias) ou vaselina salicilada a $10 \%$ (uma vez por dia durante sete dias após o banho) sem aplicar entre os dedos. Recomenda-se o uso diário de pedra-pomes nas calosidades, lixando de forma suave depois do banho. ${ }^{3,12}$ É importante averiguar a causa da hiperqueratose e corrigi-la.

O tratamento das fissuras baseia-se na aplicação diária de antissépticos suaves, como a clorohexidina ou o permanganato de potássio (se existir teci- do de granulação superficial), e de pensos hidrocolóides extrafinos durante $48 / 72$ horas. $^{12}$

Os calos deverão ser submetidos a remoção cirúrgica por profissionais com treino adequado. ${ }^{3,17,18}$ Se recorrentes, prescrevem-se ortóteses interdigitais almofadadas, com orificio central, para impedir a pressão interdigital e reforça-se a importância do calçado adequado, sem pontos de contacto anormais. Não se recomendam os agentes químicos calicidas. ${ }^{8}$

O tratamento das anormalidades ósseas é diferente consoante as características das deformações. Perante deformidades não rígidas pode-se indicar cinesioterapia, calçado adaptado mais largo e fundo e palmilhas com apoio retrocapital com ortóteses interdigitais de silicone nos dedos em garra. ${ }^{19}$ Nas deformações rígidas, além do uso de calçado adequado (incluindo sapatos à medida, embora os resultados dos trabalhos sobre este tipo de calçado sejam 
discordantes), ${ }^{17-19}$ deve-se ponderar a referenciação para ortopedia para correcção cirúrgica. ${ }^{3}$

O tratamento das flictenas não é consensual, sendo, no entanto, cada vez mais preconizada a punção das mesmas, sem remoção de restos epidérmicos e áposito, cobrindo bem a área que ajude a cicatrizar e a impedir a infecção. ${ }^{20}$

A tinea pedis pode tratar-se com antifúngicos tópicos, duas vezes por dia, durante quatro semanas. ${ }^{3}$ Deve ser evitada a humidade do pé, ensinando os diabéticos a secar bem os pés após a lavagem, sem esquecer entre os dedos. Deve-se incentivar o uso de meias de algodão. ${ }^{7}$

As unhas devem ser objecto de atenção particular. Evitam-se as unhas encravadas limando-as diariamente ${ }^{3} \mathrm{em}$ vez de as cortar. As unhas devem manter os bordos rectos. Recomenda-se o uso de calçado adequado (amplo na biqueira). ${ }^{8}$ As recidivas da unha encravada devem ser tratadas cirurgicamente. Existem estudos que confirmam a eficácia do tratamento da onicomicose com antifúngicos tópicos (cicloporoxolamina em verniz a aplicar duas vezes por semana durante dois a três meses), ${ }^{21}$ se a micose está limitada a uma ou duas unhas e sempre que a matriz não esteja atingida. É fundamental transmitir ao doente a importância de limar a unha antes da aplicação do verniz.

Em caso de opção pelos antifúngicos por via sistémica, devem ter-se em conta os seus efeitos secundários, nomeadamente a hepatotoxicidade associada ao cetoconazol e o aumento do risco de hipoglicemias em doentes que tomam imidazoles e antidiabéticos orais. ${ }^{22} \mathrm{~A}$ avulsão cirúrgica ou química será de considerar sempre que o tratamento conservador não resulta.

A vasculopatia periférica trata-se mediante o controlo apertado dos factores de risco (dislipidemia, hábitos tabágicos, controlo da glicemia e hipertensão arterial). O uso de antiagregantes está indicado. Existem estudos que evidenciam maior eficácia do clopidogrel (75 $\mathrm{mg} /$ dia per os) em relação à aspirina (375 mg/dia per os) na redução dos efeitos cardiovasculares. ${ }^{23}$

O tratamento dos sintomas de claudicação intermitente baseia-se no exercício, com eficácia comprovada do treino da marcha intermitente três vezes por semana durante um período mínimo de três meses. A pentoxifilina não tem demonstrado aumento da distância de marcha livre de claudicação. Em detrimento da pentofixilina, a American Heart Association (AHA) incluiu o cilostazol como terapêutica efectiva no tratamento dos sintomas da doença, mas esta substância ainda não está comercializada em Portugal. ${ }^{24}$ Se aparecer dor em repouso, claudicação incapacitante ou ITB menor ou igual a 0,69, deve-se referenciar aos cuidados secundários para possivel revascularização. ${ }^{23}$

O pé com sintomas de neuropatia beneficia de um controlo de glicemia apertado e do reforço dos conselhos sobre autocuidados e higiene. ${ }^{25} \mathrm{~A}$ hiperestesia pode ser tratada com amitriptilina (150 mg/dia) ou carbamazepina (máximo de $1.200 \mathrm{mg} /$ dia), associadas ou não à gabapentina (até 1.200 $\mathrm{mg} /$ dia $p e r$ os) ${ }^{9} \mathrm{ou}$ à pregabalina (máximo $300 \mathrm{mg} /$ dia).

A consulta deve ser aproveitada para realizar a educação do doente, dos familiares e cuidadores.

\section{Grau 2 - Úlcera superficial}

As úlceras devem ser submetidas a limpeza diária com soro fisiológico à pressão ou iodopovidona.

Devem-se desbridar os tecidos necróticos semanalmente com técnica cirúrgica, devendo ser moderados se o pé estiver muito isquémico (ITB $<0,5) .{ }^{11}$ Se a úlcera for neuropática e apresentar um "anel» hiperqueratósico, este deverá igualmente ser removido. Podem efectuar-se desbridamentos diários ou três 
vezes por semana utilizando geles (de $5 \mathrm{~mm}$ de espessura) ou apósitos desbridantes autolíticos ou enzimáticos, embora não existam estudos que corroborem quer a eficácia dos procedimentos químicos neste grau, quer a superioridade de uns em relação aos outros. ${ }^{24} \mathrm{No}$ tocante à aplicação de sulfadiazina argêntica, apósitos de hidrofibra, de hidrocolóides ou carvão activado, não existem estudos que corroborem a eficácia da aplicação destes produtos neste tipo de lesões. ${ }^{8}$

A analgesia efectua-se segundo a escala de dor da Organização Mundial de Saúde.

Deve realizar-se um controlo glicémico apertado, eventualmente com insulinoterapia, e compensação das doenças concomitantes. ${ }^{4}$

No tocante à oxigenoterapia hiperbárica, estimulação eléctrica e hidroterapia, não existem dados científicos que suportem a sua indicação neste grau do pé diabético. ${ }^{24}$ Por outro lado, a administração de pressão negativa intermitente parece ter eficácia no tratamento das úlceras, embora não se tenham encontrado referências de uso neste grau. ${ }^{24,25}$

O tratamento antibiótico sistémico não está formalmente indicado nesta fase, mas alguns autores, perante uma úlcera neuroisquémica, preconizam tratamento antibiótico com amoxicilina oral (500 mg três vezes/dia), flucloxacilina (500 mg quatro vezes/dia) ou eritromicina (500 mg quatro vezes/dia) depois da colheita de material para cultura da úlcera. Se a cultura for negativa a antibioterapia é retirada; se for positiva, realiza-se antibioterapia segundo o teste de sensibilidade. ${ }^{24}$

Recomenda-se repouso do membro atingido durante um período mínimo de três semanas e elevação do pé com almofada durante o sono e quando sentado. Pode-se utilizar uma fibra de vidro, trocada semanalmente, ou ortóteses, tipo bota Scotchcast ou Aircast. ${ }^{24}$ Normalmente, a fibra de vidro de con- tacto total é aplicada nas úlceras plantares que não respondem a outras medidas, ou nas úlceras recorrentes ${ }^{26} \mathrm{e} e$ é sempre necessária ajuda técnica familiarizada com a sua aplicação. ${ }^{19}$ Actualmente, embora a fibra de vidro seja vivamente recomendada para diminuir a pressão na úlcera, não tem sido muito utilizada nos cuidados de saúde, dados os seus custos, o receio de complicações e falta de profissionais treinados. ${ }^{27}$

Recomenda-se a realização de exercícios de flexo-extensão-rotação do tornozelo e joelho, já que ajudam a reduzir o edema associado à lesão.

Por último, deve avaliar-se a lesão diariamente, vigiando o aparecimento de celulite, crepitação, exsudado purulento, mau cheiro, afundamento da úlcera, fistulas e gangrena, tendo sempre em conta que os sinais e sintomas da infecção estão diminuídos no diabético. ${ }^{8,24}$ Se a úlcera não responde ao tratamento em duas semanas, o ITB é menor ou igual a 0,5 ou se há suspeita clínica que a úlcera é angiopática aguda, deve-se referenciar aos cuidados secundários. ${ }^{24}$

\section{Grau 3 - Úlcera profunda}

Os cuidados são semelhantes aos realizados nas úlceras de grau 2. Deve-se efectuar cultura com antibiograma do fundo da úlcera antes do tratamento com antibiótico. ${ }^{8,25}$ Nesta fase, as infecções, numa percentagem superior a $90 \%$, são causadas por um único organismo gram positivo, que geralmente é o estafilococo aureus ou o estreptococo.

Não existe um tratamento padrão antibiótico da úlcera ${ }^{24,25,28}$ pelo que, como antibioterapia empírica prévia ao resultado do antibiograma, se pode utilizar amoxicilina com ácido clavulâmico por via oral (500 mg 6-8 horas), flucloxacilina (500 mg quatro vezes/dia) ou ciprofloxacina (750 mg 12/12 horas). Se existir tecido necrótico ou cheiro fétido associa-se a qualquer dos anteriores a clindamicina (300 mg 6/6 horas) ou o 
metronidazol (500 mg 8/8 horas). Se o resultado do antibiograma for negativo suspende-se a antibioterapia, excepto nas situações de suspeita de isquemia severa.

O tratamento deve durar pelo menos duas semanas. ${ }^{4}$ Os antibióticos tópicos não são recomendados, excepto a mupirocina a $2 \%$ tópica, se foram isoladas estirpes de estafilococos aureus meticilino-resistente..$^{4,24,25}$

Perante lesões muito exsudativas utilizam-se produtos adsorventes, como os apósitos de hidrofibra de hidrocolóide, os alginatos ou apósitos de carvão activado. Nestas úlceras está contra-indicada a aplicação de botas de gesso.

Deve ser pedida uma radiografia do pé sempre que a úlcera não tenha boa evolução apesar do tratamento adequado, pela presença possivel de osteomielite.

\section{Grau 4 - Pé com úlcera profunda com celulite ou abcesso ou envolvimento ósseo}

Deve efectuar-se a referenciação em menos de 24 horas para os cuidados secundários, para desbridamento cirúrgico e tratamento com antibióticos endovenosos.

\section{Grau 5 - Pé com gangrena localizada}

O paciente deve ser imediatamente referenciado para cuidados secundários, para estudo urgente da circulação periférica e eventual tratamento cirúrgico (bypass, angioplastia, amputação). ${ }^{8,27}$

\section{Grau 6 - Pé com gangrena extensa}

Efectua-se referenciação urgente para cuidados secundários com vista a eventual amputação. ${ }^{8.27}$

\section{Úlceras Crónicas e Artropatia} DE CHARCOT

Não existe consenso que defina quando uma úlcera no pé do diabético pas- sa a ser crónica. Alguns autores defendem que uma úlcera que tenha tido um tratamento optimizado com mais de quatro semanas de evolução deve passar a cuidados adjuvantes, ${ }^{24}$ mas podese, por isso, determinar as quatro semanas como o tempo para definir a passagem de uma úlcera à cronicidade? As úlceras neuropáticas raramente evoluem para a cronicidade, situação mais frequente nas úlceras vasculares. ${ }^{29} \mathrm{O}$ diagnóstico diferencial entre úlcera vascular e neuropática é, portanto, fundamental. Na abordagem das úlceras crónicas devemos:

- Recomendar a lavagem diária, hidratação da pele, protecção do frio, dieta equilibrada, abandono do tabaco e realização de exercício diário.

- Controlar as doenças associadas: hipertensão, hiperglicemia e dislipidemias.

- Tratar localmente as úlceras. Em todas as úlceras do pé do diabético podem-se seguir os princípios da Agency for Health Care Policy and Research, que se resumem nestes pontos:

- Limpar as feridas no princípio do tratamento e em cada troca do apósito.

- Utilizar a menor força mecânica possivel.

- Não limpar as feridas com agentes antisépticos, como por exemplo soluções de hipoclorito de sódio, água oxigenada ou ácido acético; utilizar água e sabonete para limpar as feridas e enxaguar com soro fisiológico, secando com gaze estéril.

- Efectuar os tratamentos com pressão suficiente, que não cause trauma no leito da ferida, mas que promova a eliminação dos restos necróticos e do exsudado.

- Para estimular a cicatrização, uma vez a ferida limpa, mantém-se esta em meio húmido, para favorecer a migração celular, evitando lesar o leito recém-formado e protegendo-a de possiveis agentes infecciosos.

- Em qualquer úlcera arterial deve pro- 
teger-se a pele em volta da úlcera.

No casos de suspeita de úlcera vascular, o pilar do tratamento será a perfusão correcta da úlcera, pelo que se deve ter em conta a referenciação dos doentes para revascularização. ${ }^{29,30} \mathrm{~A}$ anticoagulação com heparina de baixo peso molecular (dalteparina 5.000 UI/ /dia subcutâneas) tem mostrado um efeito positivo na taxa de cura da úlcera, com diminuição do número de amputações. ${ }^{31,32}$ Estas úlceras beneficiam de ambiente seco no início da revascularização, mas depois deve passar-se a um ambiente húmido.

Nos casos de suspeita de úlcera neuropática, os pilares do tratamento são o desbridamento dos tecidos necróticos, a diminuição da pressão sobre a lesão e o uso de suportes ortopédicos. ${ }^{27}$

Existem múltiplas investigações para o desenvolvimento de tratamentos das úlceras. Entre as novas terapêuticas que estão a ser usadas nas úlceras neuropáticas crónicas encontram-se os factores de crescimento (factores recombinantes derivados das plaquetas tópicas, geles de colagénio e fibroblastos, ácido hialurónico e matrizes proteicas) e a bioengenharia da pele (derme artificial), produtos que estimulam a granulação e diminuem a incidência de osteomielite mas que não estão comercializadas em Portugal. ${ }^{16}$ Apresentam um preço elevado, pelo que só se aplicam em casos de úlceras que não respondem às restantes técnicas. ${ }^{24}$ No tocante à oxigenoterapia hiperbárica existem estudos que demonstram que existe uma aceleração na cura das úlceras nos pés dos diabéticos, assim como a redução do número de amputações. Não existem indicações claras de uso, mas parece razoável aplicar o tratamento de forma adjuvante em úlceras severas com risco de vida. Não existem dados científicos que suportem o uso de estimulação eléctrica e de hidroterapia neste grau do pé diabético. ${ }^{24} \mathrm{~A}$ administração de pressão negativa intermiten- te parece ter eficácia no tratamento das úlceras, melhorando a vascularização e estimulando a formação do tecido de granulação, pelo que acelera a cura. ${ }^{24.25}$ Está igualmente descrito que alguns tipos de larvas produzem enzimas proteolíticas que liquefazem os tecidos mortos ajudando a desbridar as feridas. Contudo, a utilização de larvas não é considerada, em Portugal, uma escolha no tratamento das úlceras de carácter crónico, estando esta opção relacionada com o facto de não existirem larvas disponiveis no mercado e também com sentimentos de repulsa por parte dos doentes e, muitas vezes, dos próprios profissionais de saúde. Em alguns países da Europa, em particular no Reino Unido, este tipo de tratamento é utilizado com sucesso, tendo sido, inclusivamente, desenvolvido algum trabalho na área da qualidade de vida do doente. ${ }^{33,34}$

Definimos artropatia de Charcot como uma artropatia global, com subluxação plantar do tarso, perda da concavidade interna do pé associada ou não a luxação tarsometatársica. ${ }^{35} \mathrm{Clini}$ camente aparecem pés com grandes deformações e com outras características do pé neuropático.

Esta situação exprime a fase final da artropatia diabética, que começa por fracturas espontâneas ou com traumatismo mínimo, fracturas que são por vezes indolores, mas que apresentam manifestações clínicas (pé muito edemaciado, quente e eritematoso) e radiológicas (reacção perióstea e osteólise). Está ainda associada a úlceras de tratamento dificil.

O tratamento da artropatia de Charcot é paliativo, mas deve ser o mais precoce possivel, já que evita o agravamento das deformações e das úlceras. Este tratamento passa por evitar o apoio do pé afectado, por calçado à medida e moldado às deformidades e pela vigilância e tratamento das úlceras. A amputação é por vezes necessária quando a úlcera é incurável. 
O melhor tratamento é a prevenção que assenta no diagnóstico e tratamento da fase aguda. Além das medidas aplicadas na fase crónica da doença, existem estudos que sugerem a utilidade dos bifosfonatos no tratamento. ${ }^{29}$

A abordagem do pé dos diabéticos implica uma abordagem multidisciplinar, mas perante as úlceras crónicas e a artropatia de Charcot torna-se fundamental uma comunicação excelente entre os diferentes cuidadores. O médico de familia deve coordenar activamente a situação, tanto para evitar duplicação de esforços como lacunas nos cuidados a prestar.

\section{Educaç̃o do Doente Diabítico} SOBRE OS SEUS PÉs

A educação, concebida de forma estruturada e organizada, desempenha um importante papel na prevenção das lesões do pé nos diabéticos. ${ }^{8}$ Listam-se, em seguida, as áreas que devem ser abordadas na educação do diabético, dos familiares e cuidadores:

1. Revisão diária dos pés, incluindo as áreas entre os dedos, pelo próprio ou por outra pessoa. A observação por terceiros é mandatória no caso de redução da acuidade visual. A planta do pé pode ser observada com um espelho.

2. Lavar diariamente os pés:

- Confirmar a temperatura da água com um termómetro (inferior a $37^{\circ} \mathrm{C}$ );

- Após o banho secar os pés cuidadosamente, especialmente entre os dedos;

- A lavagem deve durar aproximadamente cinco minutos.

3. Não expor os pés a temperaturas extremas, quer frias quer quentes.

4. Trocar de meias diariamente. Estas devem ser de lã ou de algodão, preferencialmente de cor clara (detecção precoce de lesão exsudativa).
5. Utilizar as meias com as costuras para fora ou de preferência sem quaisquer costuras. Não devem ter remendos.

6. Nunca caminhar descalço.

7. O calçado deve apresentar as seguintes características:

- O sapato deve medir mais um centímetro para além do dedo mais comprido;

- O tacão não deve ter mais de 2 centímetros de altura;

- O calcanhar do sapato deve ser firme;

- O dorso deve ser alto, apertado com cordões ou velcro;

- O sapato deve possuir palmilha amovivel, que seja passivel de substituição por uma palmilha individualizada;

- A troca por um novo par de sapatos deve fazer-se de modo gradual (experimentar ao fim da tarde, por exemplo);

- O material do sapato deve permitir a transpiração do mesmo (um bom material é o couro). Sapatos desportivos com sola de borracha são também uma boa escolha;

- Não utilizar calçado sem meias.

8. Inspeccionar e palpar diariamente o interior dos sapatos, antes de os calçar.

9. Não recortar as unhas excessivamente: limar é melhor do que cortar.

10. Não cortar os calos e não usar produtos químicos ou adesivos para retirar as calosidades: recorrer ao enfermeiro, médico ou podologista.

11. Se a pele estiver seca, aplicar cremes hidratantes, mas não entre os dedos.

12. Procurar caminhar diariamente.

13. Não fumar.

14. Controlar a glicemia, a pressão arterial e os lípidos.

15. Assegurar que os pés são examinados REGULARMENTE por um profissional de saúde. 
16. Informar a equipa do Centro de Saúde sobre qualquer lesão ou alteração da cor do pé.

\section{CONCLUSÃO}

Este texto tem como objectivo providenciar orientações práticas para o seguimento e tratamento dos pés dos diabéticos. Assim, salientou-se a importância do diagnóstico do pé do diabético baseado fundamentalmente nos instrumentos mais antigos da medicina: a história e o exame objectivo minucioso. Fica clara a vantagem de abordar o pé numa vertente preventiva e não apenas curativa, já que assim se melhoram os cuidados e a qualidade de vida dos pacientes diabéticos. As equipas de saúde deverão estar preparadas para a prestação de cuidados do pé diabético.

\section{REFERÊNCIAS BIBLIOGRAFICAS}

1. Paiva I, Baptista C, Ribeiro C, Leitão P, Carvalheiro $\mathrm{M}$. A diabetes na quarta idade a nossa realidade. Acta Med Port 2006 Jan-Fev; 19: (1) 79-84.

2. Bouter KP, Storm AJ, de Groot RR, Uitslager R, Erkelens DW, Diepersloot RJ. The diabetic foot in Dutch hospitals: epidemiological features and clinical outcomes. Eur J Med 1993 Apr; 2 (4): 215-7.

3. Grupo Diabetes SAMFyC. Pie diabético. Disponivel em: URL: http://www.cica.es/ sa mfyc/pie.htm [acedido em 22/12/2006].

4. Direcção Geral de Saúde. Pé diabético Programa de controlo da diabetes mellitus. Circular Normativa n. ${ }^{\circ}$ 8. Lisboa: DGS; 2001.

5. Lowrie WL, Redfern WE, Brush BE. The diabetic foot. Clin Orthop 1955; 6: 173-81.

6. Duque FL, Duque AC. Considerações sobre o termo pé diabético. Rev Angiol Cir Vasc 2001; 10 (4): 158-60.

7. S/A. Consenso sobre pie diabético. Angiologia 1997; 49 (5): 193-7.

8. Sociedade Portuguesa de Diabetologia; Grupo de Trabalho Internacional sobre o Pé Diabético. Directivas Práticas sobre o Tratamento e a Prevenção do Pé Diabético. Lisboa: Bayer Portugal; 1999.

9. Gross JL, Nehme M. Detecção e tratamento das complicações crônicas do diabetes meli- to: Consenso da Sociedade Brasileira de Diabetes e Conselho Brasileiro de Oftalmologia. Rev Ass Med Brasil 1999; 45 (3): 279-84.

10. Boyko EJ, Ahroni JH, Cohen V, Nelson KM, Heagerty PJ. Prediction of diabetic foot ulcer occurrence using commonly available clinical information: the Seattle Diabetic Foot Study. Diabetes Care 2006 Jun; 29(6): 1202-7.

11. Edmonds ME, Foster AV. ABC of wound healing: diabetic foot ulcers. BMJ $2006 \mathrm{Feb} 18$; 332 (7538): 407-10.

12. Tizon Bouza E, Dovale Robles MN, Fernández García MY, Fernández Veiga C, López Vilela M, Mouteira Vázquez M, et al. Atención de enfermería en la prevención y cuidados del pie diabético. Aten Primaria 2004 Sep; 34 (5): 263-5 .

13. American Diabetes Association. Peripheral arterial disease in people with diabetes. Diabetes Care 2003 Dec; 26 (12): 3333-41.

14. Oyibo SO, Jude EB, Tarawneh I, Nguyen HC, Harkless LB, Boulton AJ. A comparison of two diabetic foot ulcer classification systems: the Wagner and the University of Texas wound classification systems. Diabetes Care 2001 Jan; 24 (1): 84-8.

15. Rooh-Ul-Muqim, Ahmed M, Griffin S. Evaluation and management of diabetic foot according to Wagner's classification: a study of 100 cases. J Ayub Med Coll Abbottabad 2003 Jul-Sep; 15 (3): 39-42.

16. Asociación Zamorana de Traumatología y Cirugia Ortopédica (AZACOT). Autocuidados del pie diabético. Disponivel em: URL: http:// www.traumazamora.org/infopaciente/ piediabetico.html [acedido em 22/03/2007].

17. Mayfield JA, Reiber GE, Sanders LJ, Janisse D, Pogash LM; American Diabetes Association. Preventive foot care in people with diabetes. Diabetes Care 2003 Jan; 26 Suppl 1: S78-9.

18. American Diabetes Association. Standards of medical care in diabetes - 2006. Diabetes Care 2006 Jan; 29 Suppl 1: S4-42.

19. García I, Zambudio R. Ortesis, calzados y prótesis. In: Roura JM, Mompó JI, Rodríguez JR, Esquembre VI, Olay JR, editores. Tratado de pie diabético. Madrid: Jarpyo Editores; 2002. p. 139-53. Disponivel em: URL: http:// www.esteve.es/EsteveArchivos/1_8/Ar_1_8_42_ APR_6.PDF [acedido em 10/05/2007].

20. Petit JM. Flictenas. Disponivel em: URL: http://www.podologo.cl/index.php? Option $=$ com_content $\&$ task $=$ view\&id $=19 \& I t e m i d=30$ [acedido em 11/01/2007].

21. Seebacher C, Nietsch KH, Ulbricht HM. A multicenter, open-label study of the efficacy and safety of ciclopirox nail lacquer solution $8 \%$ for the treatment of onychomycosis in patients with diabetes. Cutis 2001 Aug; 68 (2 Suppl): 17-22. 


\section{DOSSIER}

22. Robbins JM. Treatment of onychomycosis in the diabetic patient population. J Diabetes Complications 2003 Mar-Apr; 17(2):98-104.

23. Edmonds M. Diabetic foot ulcers: practical treatment recommendations. Drugs 2006; 66 (7): 913-29.

24. Hirsch AT, Haskal ZJ, Hertzer NR, Bakal $\mathrm{CW}$, Creager MA, Halperin JL, et al. ACC/AHA 2005 Practice Guidelines for the management of patients with peripheral arterial disease (lower extremity, renal, mesenteric, and abdominal aortic): a collaborative report from the American Association for Vascular Surgery/Society for Vascular Surgery, Society for Cardiovascular Angiography and Interventions, Society for Vascular Medicine and Biology, Society of Interventional Radiology, and the ACC/AHA Task Force on Practice Guidelines (Writing Committee to Develop Guidelines for the Management of $\mathrm{Pa}$ tients With Peripheral Arterial Disease): endorsed by the American Association of Cardiovascular and Pulmonary Rehabilitation; National Heart, Lung, and Blood Institute; Society for Vascular Nursing; TransAtlantic Inter-Society Consensus; and Vascular Disease Foundation. Circulation 2006 Mar 21; 113 (11): e463-654.

25. Boulton AJ, Kirsner RS, Vileikyte L. Clinical practice: neuropathic diabetic foot ulcers. N Engl J Med 2004 Jul 1; 351 (1): 48-55.

26. Nabuurs-Franssen MH, Huijberts MS, Sleegers R, Schaper NC. Casting of recurrent diabetic foot ulcers: effective and safe? Diabetes Care 2005 Jun; 28 (6): 1493-4.

27. Singer AJ, Clark RA. Cutaneous wound healing. N Engl J Med 1999 Sep 2; 341 (10): 738-46.

28. Pitocco D, Ruotolo V, Caputo S, Mancini L, Collina CM, Manto A, et al. Six-month treatment with alendronate in acute Charcot neuroarthropathy: a randomized controlled trial. Diabetes Care 2005 May; 28 (5):1214-5.

29. Katz IA, Harlan A, Miranda-Palma B,
Prieto-Sanchez L, Armstrong DG, Bowker JH, et al. A randomized trial of two irremovable offloading devices in the management of plantar neuropathic diabetic foot ulcers. Diabetes Care 2005 Mar; 28 (3): 555-9.

30. Consensus Development Conference on Diabetic Foot Wound Care: 7-8 April 1999, Boston, Massachusetts. American Diabetes Association. Diabetes Care 1999 Aug;22(8):1354-60.

31. Rooke TW. Dalteparin improved chronic foot ulcers and reduced the number of amputations in diabetic peripheral arterial occlusive disease. Evid Based Med 2004 May-Jun;9(3): 73.

32. Kalani M, Apelqvist J, Blomback M, Brismar K, Eliasson B, Eriksson JW, et al. Effect of dalteparin on healing of chronic foot ulcers in diabetic patients with peripheral arterial occlusive disease: a prospective, randomized, doubleblind, placebo-controlled study. Diabetes Care 2003 Sep; 26 (9): 2575-80.

33. Elias CG. Tratamento de feridas crónicas com larvas. Disponivel em: URL: http:// www.gaif.net/artigos/larvas.pdf [acedido em 23/03/2007].

34. Sherman RA. Maggot therapy for treating diabetic foot ulcers unresponsive to conventional therapY. Diabetes Care 2003 Feb; 26 (2): 446-51.

35. Grupo de Trabajo sobre Úlceras Vasculares de la AEEV. Consenso sobre las úlceras vasculares y pie diabético de la Asociación Española de Enfermeria Vascular. 2004. Disponível em: URL: http://www.aeev.net/ documentos/consenso2005.pdf [acedido em 22/03/ /2007].

\section{Endereço para correspondência}

Gema Ponce Revilla

Rua Artur Lamas, 1-3ํㅡㄹ Esq.

1300 Lisboa

E-mail: gema_ponce@yahoo.es

\section{THE DIABETIC FOOT}

\section{ABSTRACT}

Introduction: Problems arising in the feet of diabetic patients are a critical aspect of their care, both in terms of frequency and consequences. Our aim in this review is to establish practical recommendations for the treatment and preventive of this problem in primary care.

Methods: We searched the Pubmed database for articles and reviews on the diabetic foot. We also used standard reference texts.

Conclusion: A simple staging system is used to evaluate the risk of development of diabetic foot injuries and to define appropriate screening periods. Management recommendations are made based on a risk classification and the presence of ulceration.

Keywords: Diabetic Foot; Neuropathic Foot; Ischemic Foot; Diabetic Foot Ulcer; Foot at Risk; Screening of the Diabetic Foot; Prevention. 